\title{
Properties of heat-sealing BOPP film: effect of coating thickness and random copolymer content
}

\author{
Jie $\mathrm{Du}^{1,}$, , Feng $\mathrm{Yu}^{1}$, Shuna Meng ${ }^{1}$, Hongcun Huang ${ }^{2}$ \\ ${ }^{1}$ College of Materials and Chemistry Engineering, Hainan University, Haikou 570228, China \\ ${ }^{2}$ Sainuo Industrial Co., Ltd., Haikou 570125, China \\ *dujie@hainu.edu.cn
}

\begin{abstract}
Keywords: heat -sealing strength; temperature; thickness; friction coefficient; random copolymer
Abstract. Nowadays heat-sealing biaxial oriented polypropylene (BOPP) films are widely used as packages because of its size-stability and nontoxicity. However, the heat sealing strength is relatively low, which makes BOPP films not satisfy the increasing performance as the modern packagings. Therefore, an effective research on the relationship of the properties of BOPP film and its physical parameters is in great need. In this paper, we tested heat-sealing strength of BOPP films with different surface coating thickness and with different compositions. The results showed that the heat sealing strength of BOPP films increased continuously and slowly with increasing temperature. At the same heat sealing temperature, the heat sealing strength significantly changed with the coating thickness. From random copolymer to mixture, the range of suitable heat sealing temperature gradually increased.
\end{abstract}

\section{Introduction}

The heat sealing strength of the plastic layers is the same or different materials fills the heat-bonded together at a certain temperature and pressure, and then the peel strength is reached. Biaxial oriented polypropylene (BOPP) is a very important product in the package field [1-3]. It is a kind of waterproof and high mechanical specification material, and it is size-stabled and very light without any smell and toxicity. But usually its heat sealing strength is generally only $10 \mathrm{~N} / 15 \mathrm{~mm}$, lower than the extensive use of composite membrane or coating film in the market.

To achieve a single layer environmental film instead of the multilayer composite membrane or coating film, we must further improve its heat-sealing strength through a study on the relationship between the structure and function of materials. This project aims at the systematic study of the composition and structure of sealing materials for films, to establish a composition - structure function relationship, and examine how the seal strength, haze and friction coefficient change with the variation of the coating thickness.

\section{Experimental}

Material and techniques. Brugger HSG-C Heat seal tester (Beijing Dan Beier Instrument Co., Ltd); WGT-S Transmittance haze meter (Beijing Science and Technology Co., Ltd. baiyuan Ark); MXD-01 Coefficient of friction tester (GREAT Ling Technology Co., Ltd. in Jinan).

These co-extruded films were made with three layers. One central layer of homopolymer of polypropylene (i-PP) $(95.5 \%)$. The two external layers were manufactured using a random copolymer of poly propylene and 3\% of polyethylene-polybutylene as rawmaterial. Some additives, as antiblocking and slipagents, were added in quantities less than $1000 \mathrm{ppm}$. The core layer represents $90 \%$ and the skin layers $10 \%$ of the film. This compounding was confirmed by micro thermal analysis. 


\section{Results and Discussion}

\section{Heat sealing strength changing with the thickness and temperature}

Three-layer (represented by A, B, C) BOPP films were manufactured with the above methods. Surface A was coated with polyvinyl alcohol, surface $C$ was coated with acrylic, and surface B is BOPP film (Thickness of 20um). The coating thicknesses are $0.8 \mathrm{um}, 1.0 \mathrm{um}, 1.2 \mathrm{um}, 1.5 \mathrm{um}, 1.8 \mathrm{um}$, and $2.0 \mathrm{um}$, respectively. We used Brugger HSG-C heat sealing tester to detect how the heat-sealing strengths of $\mathrm{A} / \mathrm{A}, \mathrm{A} / \mathrm{C}$, and $\mathrm{C} / \mathrm{C}$ interfaces change with temperature and coating thickness (Fig. 1). The heat sealing strength on $\mathrm{A} / \mathrm{A}$ interface increases obviously with increasing temperature below $120{ }^{\circ} \mathrm{C}$, while has a little change above $120{ }^{\circ} \mathrm{C}$. At the same heat-sealing temperature, the heat sealing strength increases with higher coating thickness. The heat sealing strength on $\mathrm{A} / \mathrm{C}$ interface increases significantly till $110^{\circ} \mathrm{C}$, while increases gradually between $110^{\circ} \mathrm{C}$ and $120^{\circ} \mathrm{C}$, and decreases above $120^{\circ} \mathrm{C}$. Similar trend of the heat sealing strength with different coating thickness as A/A interface is also showed in Fig. 2. The increasing rate of heat sealing strength on $\mathrm{C} / \mathrm{C}$ interface is obviously weakened below $105{ }^{\circ} \mathrm{C}$. As the temperature increases, the heat sealing strength increases continuously and slowly. At the same heat sealing temperature, the heat sealing strength of BOPP significantly changed with the coating thickness.

\section{The thickness and friction coefficient}

To investigate the relation of thickness and friction coefficient, we measured the dynamic/static friction coefficient of the interfaces with different coating thickness (Fig. 2). We used I/O/M to represent different coated interfaces (polyvinyl alcohol surface, acrylic surface and metallic surface, respectively). From the curve trend of friction coefficient with the increasing thickness, we can see that the thickness does not affect the coefficient of static friction. The changes of coefficient of static friction and coefficient of dynamic friction with coating thickness have the similar trends.

\section{Heat sealing strength changing with random copolymer content}

We changed the raw material of the external layers to study the effect of the random copolymer content on heat-sealing properties. Three kinds of compositions were used in this study: (1) a random copolymer of polypropylene and $1 \%$ of polyethylene, (2) a random copolymer of polypropylene and $3 \%$ of polyethylene-polybutylene, (3) mixture of 2 and 3\% polybutylene. As shown in Fig. 3, the suitable heat sealing temperature range of the mixture is the broadest.

\section{Conclusions}

We tested various performance indicators of heat-sealing BOPP films with different surface coating thickness in this paper. The results show that with the same coating thickness of BOPP films, the heat-sealing strength of $\mathrm{A} / \mathrm{A}$ and $\mathrm{C} / \mathrm{C}$ interfaces increase with increasing temperature. At the same temperature, the heat sealing strength increases with increasing coating thickness. As the film coating thickness increases from different heat sealing interfaces, it exposures a little impact on the friction coefficient, and the changes of static and dynamic coefficient friction display the similar trends. From random binary copolymer to mixture, the range of suitable heat sealing temperature gradually increased.

\section{Acknowledgements}

This work was financially supported by Natural Science Foundation of Hainan Province of China (512111).

\section{References}

[1] M. B. Elias, R. Machado, S. V. Canevarolo. J. Therm. Anal. Cal., Vol. 59 (2000), 143-155.

[2] F. Sadeghi, P. J. Carreau. Can. J. Chem. Eng., Vol. 86 (2008), 1103-1110.

[3] Y. Koike, M. Cakmak. Macromolecules, Vol. 37 (2004), 2171-2181. 

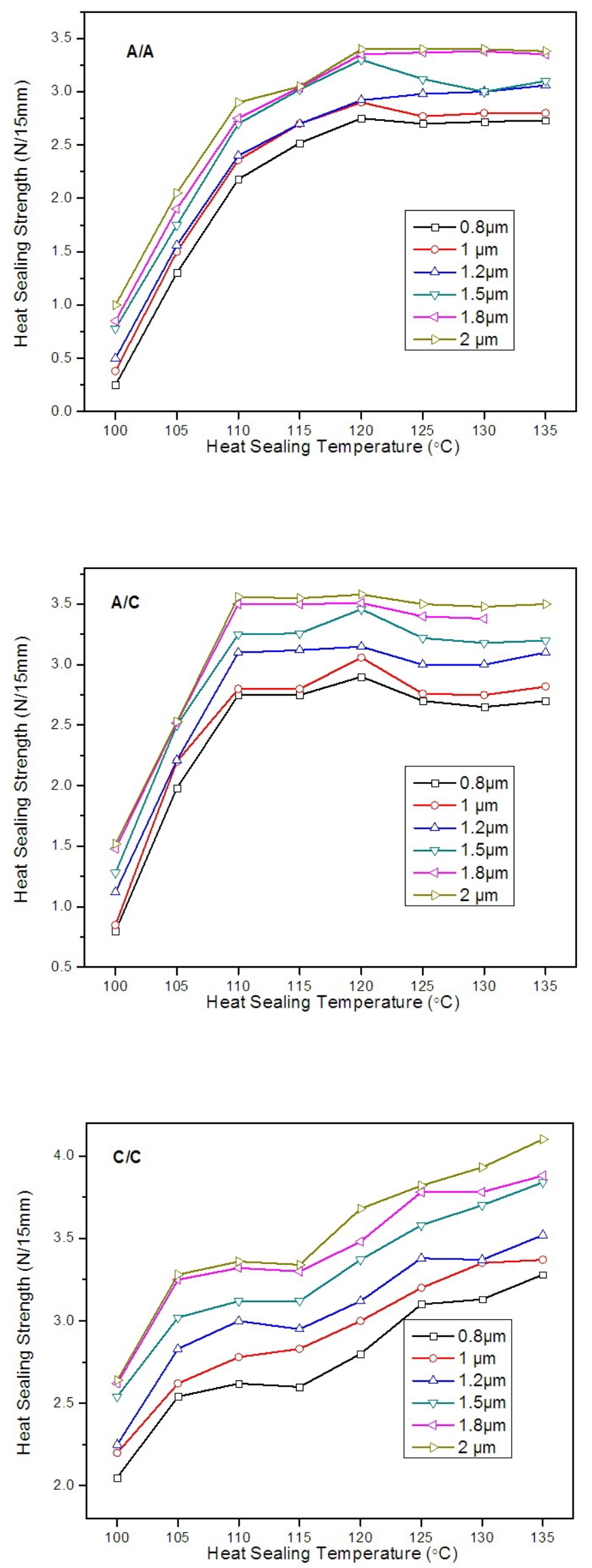

Fig. 1. Heat sealing strength of BOPP with different thickness. 

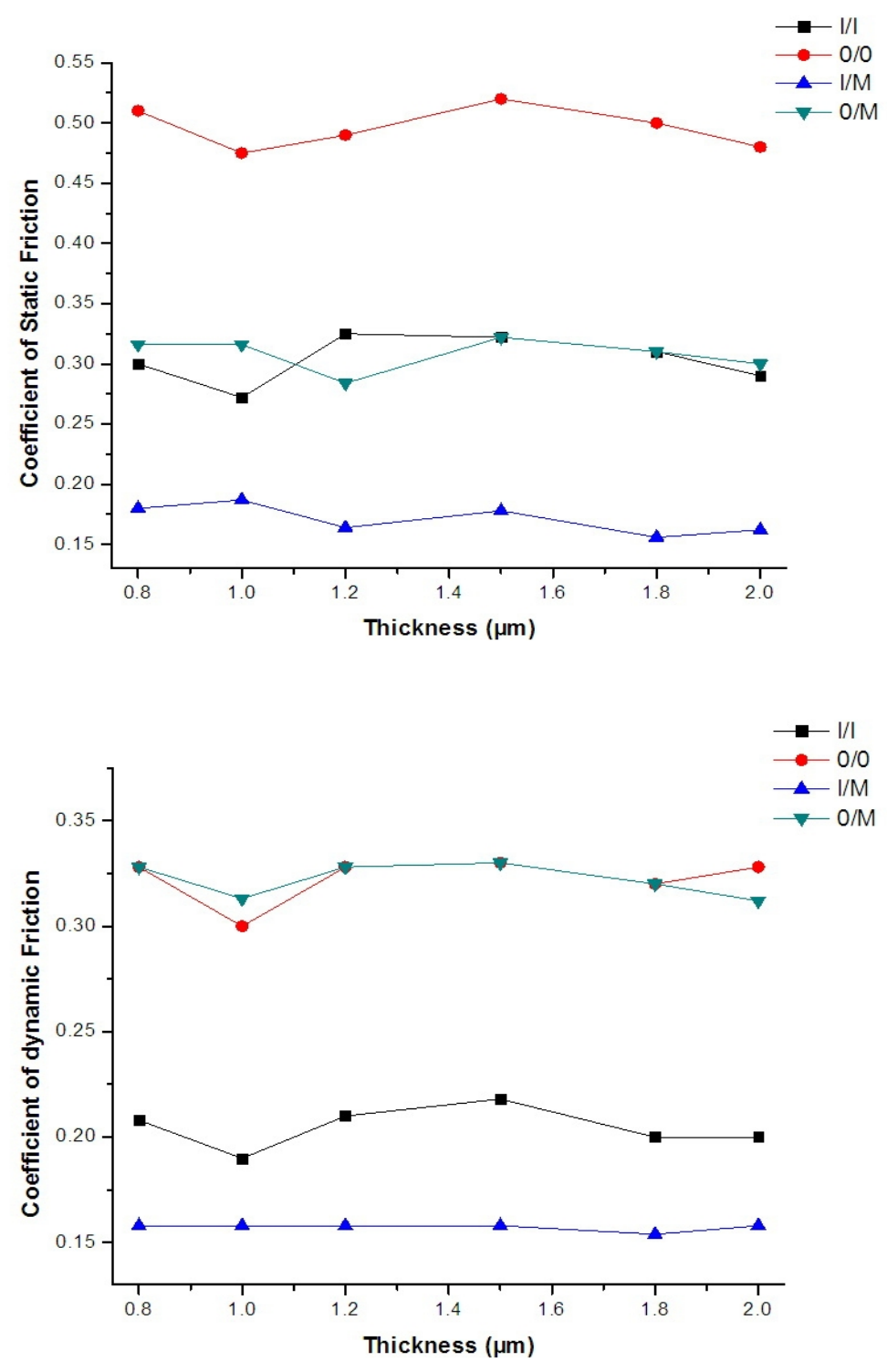

Fig. 2. Coefficient friction of dynamic/static of the two curves with thickness curves.

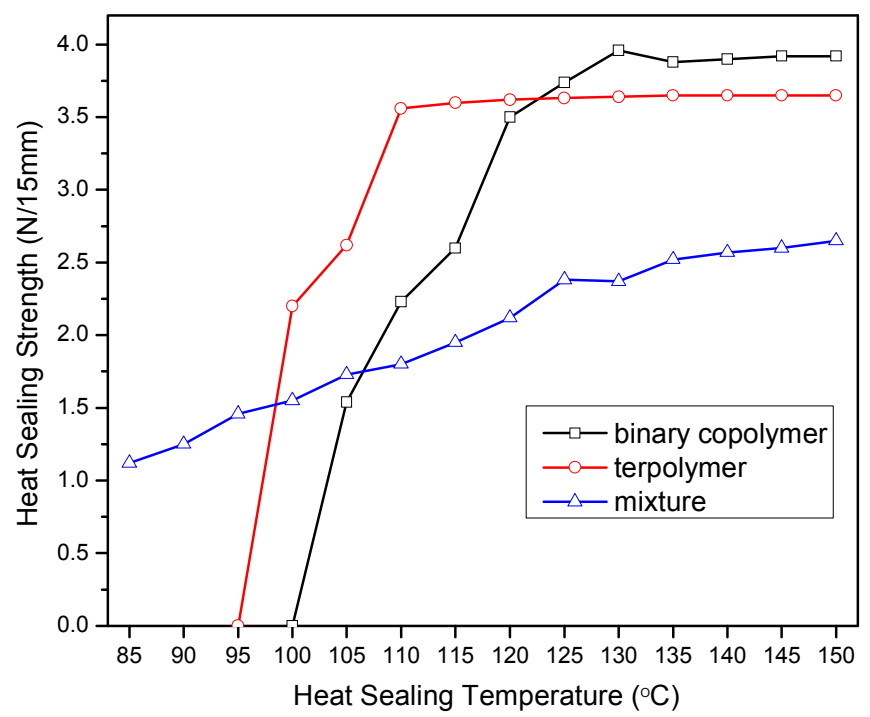

Fig. 3. Heat sealing strength of BOPP with different copolymer contents. 